\title{
26465 - DOES STAGING BILATERAL KNEE SURGERY DECREASE CARDIAC MORBIDITY?
}

\section{SummerSyed MD, Tomas Vanhelder, MD PhD; Duminda Wijeysundera, MD; , ; , Toronto General Hospital, UHN, Toronto, ONTARIO, Canada}

INTRODUCTION: Joint arthroplasty is associated with high morbidity. The optimal management strategy for patients requiring bilateral knee arthroplasty remain unclear. Replacement of both knees simultaneously may be a more cost effective strategy because it involves a single surgery, anesthetic, and rehabilitative period (1). Disadvantages include longer surgical time, increased blood loss, and higher cardiovascular morbidity $(2,3)$. Among patients known to require bilateral knee surgery, it remains to be determined which strategy is superior (4).

METHODS: Following REB approval, the charts of 2209 consecutive patients (1995 1996, 1998 - 2001) at a single institution were retrospectively reviewed. The sub-group of patients who underwent primary simultaneous bilateral knee surgery or staged bilateral knee surgery $(n=359)$ were identified. Unadjusted comparison of the two groups was made with regards to baseline characteristics and total complications. Multivariable logistic regression was employed to determine the adjusted association between surgical strategy and risk for cardiovascular complications.

RESULTS: Of the 359 patients having bilateral surgery 247 had simultaneous knee surgery and 56 had staged procedures (112 surgeries). Baseline characteristics were similar between groups. The staged group had a higher ASA classification $(\mathrm{p}<0.001)$. Length of stay and BMI were higher in the simultaneous group $(\mathrm{p}<0.001, \mathrm{p}=0.04)$. Simultaneous surgeries had a higher total complication rate per surgery $(\mathrm{p}=0.008)$; however, when the risk per person was calculated, the two had similar rates $(\mathrm{p}=0.44)$ (Figure). In multivariable analysis the staged procedure was associated with a significantly lower risk of cardiovascular complications (odds ratio 0.29, $\mathrm{p}=0.006$ ); however, when compared as a per person risk, this association lost statistical significance (odds ratio $0.70, \mathrm{p}=0.45$ ).

DISCUSSION: The choice between simultaneous bilateral and staged bilateral surgery should be made based on patient and surgeon preference. There is no significant difference in morbidity between these two strategies. Patients undergoing a staged procedure have a decreased risk per procedure, but experience a similar cumulative risk when both separate procedures are accounted for. A possible limitation to the analysis is that we could not account for patients who had dropped out between planned staged procedures for any reason.

REFERENCES: 1.Clin Orthopaed rel res (1994); 309: 94-101, 2. CJA (2005); 52:A58. 3. J bone Joint surg (2003); 85A(10): 1981-1986, 4. J Bone Joint Surg (2003)85:1532-1537. 


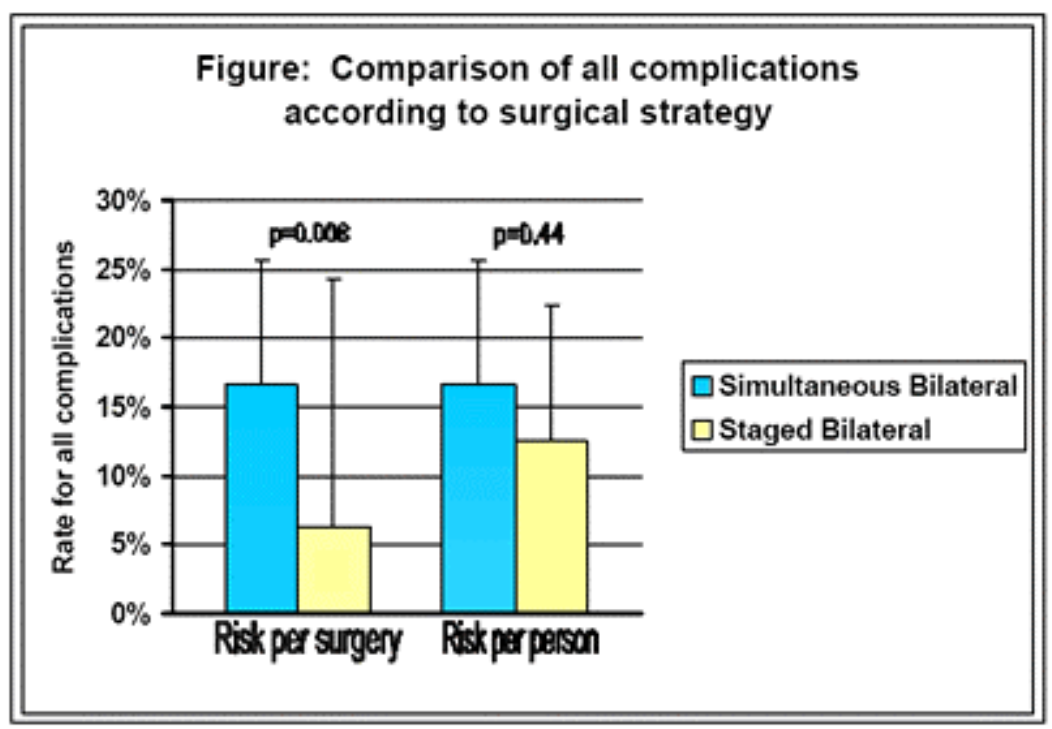

